\title{
An Improved Fractal Coding Method based on K-means Clustering
}

\author{
Hui Guo \& Jie He \\ Guangxi Colleges and Universities Key Laboratory of Image Processing and Intelligent Information System, \\ Wuzhou University, Wuzhou543002, Guangxi, China
}

\begin{abstract}
This paper focuses on a fast fractal coding algorithm based on k-means clustering. First of all, the variance method is employed to divide the sub-blocks into simple sub-blocks and complex sub-blocks; then, the k-means clustering algorithm is applied to classify the complex sub-blocks and father blocks, and the approach of nearest neighbor search is applied in the process of searching for matching father blocks, so as to match corresponding sub-blocks with father blocks of the same type only within the neighboring scope. This method optimizes the process of searching for matching blocks, thereby greatly shortening the encoding duration. Test results show that compared with the basic fractal coding algorithm, this method can increase the encoding speed by about 570 times, and lead to high quality of the reconstructed image.
\end{abstract}

KEYWORD: Fractal image coding; k-means clustering; nearest neighbor search; variance method

\section{INTRODUCTION}

Despite its advantages of novel ideas, independence from resolution, large decoding speed, high compression ratio, etc., fractal image coding algorithm can hardly achieve real-time encoding due to the large computational complexity in encoding and long compressing duration. Therefore, the enhancement of fractal coding speed has become one of the major directions in the research of fractal image compression. There are currently two types of improvements of the fractal coding algorithm, namely, sub-block classification and neighborhood search.

Sub-block classification means classifying subblocks and parent blocks according to certain characteristic before searching for matching blocks, so as to substitute global search for intra-class search during the matching process, thereby enhancing the encoding speed. A lot of attempts have been made by scholars at home and abroad in recent years to design accurate classification methods. According to Literature (Yi Chuanjun et al, 2015), the edge classification algorithm can be applied to separate parent blocks into edge ones and non-edge ones, and all types of parent blocks can be sequenced according to the average deviation. Literature (Wang Qiang et al, 2011) proposes a fast encoding algorithm based on relevant information features: the sub-blocks are partitioned into two categories according to relevant information features, so as to convert the sub-block search issue into the issue of searching for the same type of adjacent parent blocks based on relevant information features. Literature (Wang Xiang-Yang et al, 2008) puts forward a method, which is based on mean-standard deviation, for selecting the initial cluster center in k-means clustering, and uses it for clustering sub-blocks and parent blocks in fractal image coding. Literature (K. Jaferzadeh et al, 2012) adopts the pixel value space and $1 D$-DCT vector to realize fuzzy clustering, thus enhancing the encoding speed by 40 times without the loss of encoding quality.

A large number of experimental data demonstrate that parent blocks that match sub-blocks are mostly adjacent to the sub-blocks, thus neighborhood search has become the most studied optimization method in recent years. Literature (Y.-L.Lin et al, 2011) realizes neighborhood search by taking advantage of the phenomenon that blocks with similar edge shapes tend to concentrate in certain specific areas. Continuous progress is obtained in Literatures (Li Gaoping, 2011) (Li Gaoping et al, 2011) (Li Gaoping et al, 2013): they successively use the three-mean feature, four-digit feature and rotational inertia feature to implement the neighborhood search method. In addition, Literatures (Sheng Fei, 2013) (Yang Pei, 2013) propose neighborhood search methods respectively based on similarity ratio and relative error.

In this paper, in light of the above two improvement schemes, the variance-based classification method is first applied to divide sub-blocks into simple sub-blocks and complex sub-blocks, and only 
complex ones are encoded; then, the method presented by Literature (Wang Xiang-Yang et al, 2008) is used to select the initial cluster center and cluster sub-blocks and parent blocks; and then, the nearest neighbor search method is employed in the process of searching for matching father blocks, so as to match corresponding sub-blocks with only neighboring parent blocks of the same type. Experimental results show that: compared with the basic fractal image coding algorithm, the algorithm presented in this paper (hereinafter referred to as "this Algorithm") can increase the encoding speed by more than 570 times while ensuring the quality of the reconstructed image; compared with the algorithm presented in Literature (Wang Xiang-Yang et al, 2008), this Algorithm is 190 times faster while ensuring the quality of the reconstructed image.

\section{THE BASIC FRACTAL IMAGE CODING}

In the basic fractal image coding, an image is first segmented into a set of non-overlapping sub-blocks (hereinafter referred to as " $R$-blocks") with a size of $B \times B$; then, with $\lambda$ as step length, a $2 B \times 2 B$ window moves from left to right and from top to bottom to generate the set of parent blocks (hereinafter referred to as " $D$-blocks"). Subsequently, the $D$-blocks undergo the operation of four-neighborhood pixel averaging, and the generated $D$-block set is used as the matching operation codebook $\Omega$ which then undergoes eight kinds of isometric transformations to expand itself. For any $R$-block, the best matching parent block $D_{m}$ that can satisfy Formula (1) is searched for.

$$
E(R, D)=\min _{m}\left\{\min _{s, o}\left\|R-s D_{m}-o I\right\|^{2}\right\}
$$

In Formula (1), $s$ and $o$ represent the grayscale translation parameter and contrast adjustment parameter, respectively. Firstly, the values of $s$ and $o$ that can minimize the interior of the above formula are calculated, as shown in Formula (2); secondly, the global search method is used to search the expanded codebook $\Omega$ for the parent block $D_{m}$ that can minimize the exterior of Formula (1). The above operation is repeated for every $R$-block. Meanwhile, the upper-left corner coordinates $(x, y)$, isometric transformation parameter $n$, grayscale translation parameter $s$ and contrast adjustment parameter $o$ of the corresponding matching block $D_{m}$ are recorded, before the encoding of the whole image is completed. $|s|<1$ is theoretically required so as to ensure the convergence in iterative decoding, and $s$ values which do not meet the requirements are truncated.

$s=\frac{\langle R-\bar{R} I, D-\bar{D} I\rangle}{\|D-\bar{D} I\|^{2}}, o=\bar{R}-s \cdot \bar{D}$
By combining Formula (1) with Formula (2), the calculation formula for $E(R, D)^{2}$ is obtained, as shown in Formula (3):

$$
E(R, D)^{2}=\|R-R I\|^{2}-s^{2}\|D-D I\|^{2}
$$

Where, 13 is the vector inner-product computation, $\|\cdot\|$ is the vector 2-norm, and $I$ represents the matrix entirely of 1 .

In the process of decoding, an arbitrary initial image is taken; and for each $R$-block, according to corresponding $D$-block information in the encoding record, the $D$-block is taken from the specified location and goes through the processes of fourneighborhood pixel averaging, isometric transformation as well as contrast and grayscale adjustment, before corresponding values are assigned to this $R$ block. One operation is conducted for each $R$-block until an iteration of the whole image is finished, and then, by regarding the post-iteration image as the initial image, multiple iterations are conducted until the completion of the decoding.

\section{THE VARIANCE-BASED CLASSIFICATION METHOD}

In the basic fractal coding method, the whole codebook $\Omega$ has to be searched to find the best matching $D$-block for each $R$-block, which is very timeconsuming. To solve this problem, image blocks can be classified prior to fractal coding. In this paper, the method of variance is applied to classify all the $R$ blocks into two categories, namely, simple subblocks and complex sub-blocks, and only complex sub-blocks are encoded; for a simple sub-block, the mean pixel value and the coordinates of its upper left corner are stored. In decoding, the mean pixel value is used for filling in the block. This method avoids the encoding of simple sub-blocks, decreases the encoding duration and improves the compression ratio. Formula 4 below is the formula for computing the variance of $R$-block.

$$
R_{\mathrm{var}}=\sqrt{\frac{1}{n}\left(\sum_{i}^{n} r_{i}^{2}\right)-\left(\frac{1}{n} \sum_{i}^{n} r_{i}\right)^{2}}
$$

The selection of variance threshold has a large impact on the performance parameters of fractal image coding: a larger variance threshold will lead to a smaller number of complex sub-blocks, shorter encoding duration, and also lower quality of the reconstructed image. Test is made on multiple images in this paper, so as to analyze the relationships between variance threshold and various performance parameters. Figure 1 and Figure 2 below separately show the relationship between variance threshold and encoding duration as well as between variance threshold and peak signal-to-noise ratio (PSNR) of the reconstructed image. 


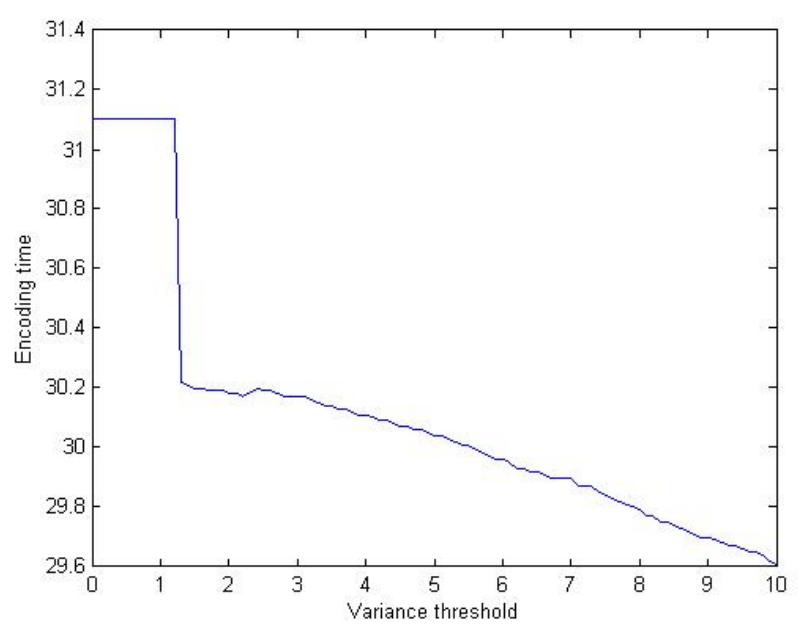

Fig. 1 The relationship between variance threshold and PSNR

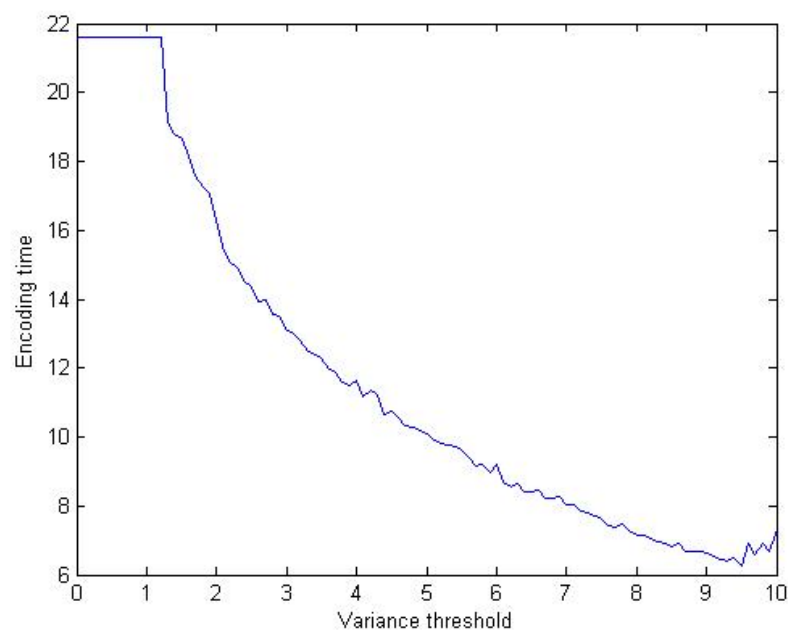

Fig. 2 The relationship between variance threshold and encoding duration

According to Figures 1 and 2, when the variance threshold is 5, PSNR of the reconstructed image is still above 30, but the encoding duration is significantly cut down. Based on comprehensive consideration of various parameters, 5 is selected as the variance threshold herein, then $R$-blocks are divided into simple sub-blocks $\left(R_{\mathrm{var}}<=5\right)$ and complex subblocks $\left(R_{\text {var }}>5\right)$, and only complex sub-blocks are encoded.

\section{NEAREST NEIGHBOR SEARCH}

According to a lot of statistical data, there is a large probability that the best matching block for the $R$ block to be encoded is in its neighborhood, as shown in Figure 3. Hence, when searching for the best matching block for the $R$-block to be encoded, the search scope can be greatly narrowed by searching its neighborhood.

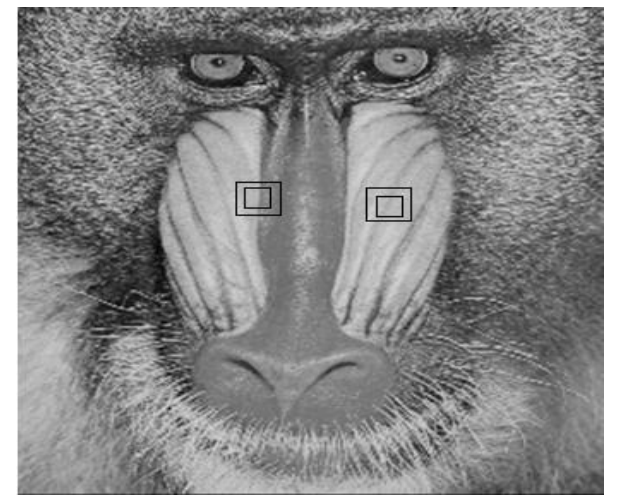

Fig. 3 The best matching block in the neighborhood

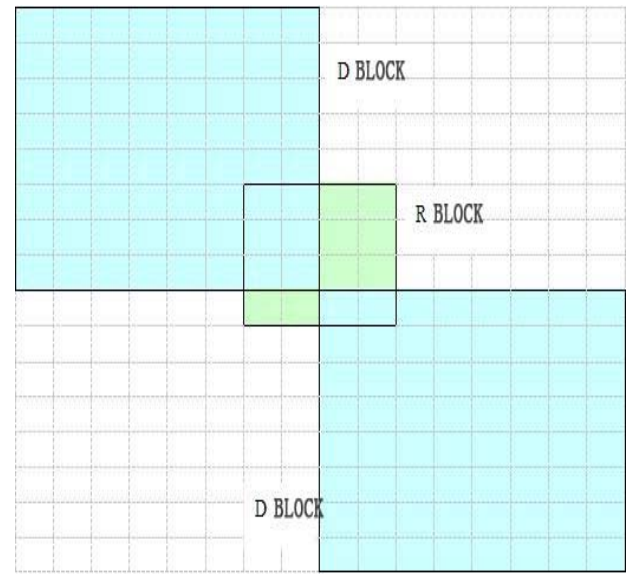

Fig. 4 R-block nearest neighbor search

In order to reduce the number of matching blocks in the search, a scope is selected near the $R$-block to be encoded, and then the search for the best matching block is conducted within this scope, as shown in Figure 4.

According to Figure 4, the scope of the search for $D$-block can be defined around $R$-block. Let $R X$ be the length of $R$-block, $D X$ be the length of $D$-block and $D X / 4$ be the step length here, the definition domain blocks are scanned at the upper left corner, lower left corner, upper right corner and lower right corner of $R$-block; since the size of the image is $N \times$ $N$, the scan will go beyond the scope at the right end, thus the number of the definition-domain blocks in horizontal scan is set to $h-R X$, in which $h$ is the square root of the total number of $D$-blocks. By the same token, the numbers of blocks in vertical scan is equal to that in horizontal scan. Therefore the scope of the blocks in the search is:

$$
\begin{aligned}
& a=\frac{4 \times(i \times R X)}{D X}-\frac{h+R X}{16} \\
& b=\frac{4 \times(i \times R X)}{D X}+\frac{h+R X}{16} \\
& c=\frac{4 \times(j \times R X)}{D X}-\frac{h+R X}{16}
\end{aligned}
$$


$d=\frac{4 \times(j \times R X)}{D X}+\frac{h+R X}{16}$

In Formulas 5-8, $i$ represents the $x$ coordinate of the $R$-block, $j$ represents they coordinate of the $R$ block, $a$ is the number of $D$-blocks searched for in the upper left corner of the $R$-block, $b$ is the number of $D$-blocks searched for in the lower left corner of the $R$-block, $c$ is the number of $D$-blocks searched for in the upper right corner of the $R$-block, and $d$ is the number of $D$-blocks searched for in the lower right corner of the $R$-block.

If $D$-block is not found in the upper left corner of $R$-block, then set a scope, start the search from $D(i$, $j$ ), and set $\mathrm{a}=1$; if $a<1$, also set $a=1$. If $D$-block is not found in the upper right corner of $R$-block, then set a scope, search until $D(i, h)$, and set $b=h$; if $b>h$, also set $b=h$. If $D$-block is not found in the lower left corner of $R$-block, then set the scope, start the search from $D(i, j)$, set $c=1$; if $c<1$, also set $c=1$. If $D$ block is not found in the lower right corner of $R$ block, then set the scope, search until $D(h, j)$, set $d$ $=h ;$ if $d>h$, also set $d=h$.

\section{THE IMPROVED FRACTAL IMAGE CODING METHOD BASED ON K-MEANS CLUSTERING}

There are mainly two reasons contributing to the long duration of fractal coding: one is the excessively large codebooks search domain formed by $D$ blocks and the other is the application of the global search method to the search for the best matching block. The currently popular solutions to these questions are: to use the classification method to settle the problem of excessively large search domain, and to substitute the global search approach for the nearest neighbor search approach. In this paper, on the basis of Literature (Wang Xiang-Yang et al, 2008), an improved fractal image coding method based on k-means clustering is put forward to settle these two problems.

1. The principle of $\mathrm{k}$-means clustering algorithm

As a partition-based type of clustering method, kmeans clustering algorithm has been widely applied. Image segmentation by using k-means clustering algorithm features large speed, good effect and high applicability. K-means clustering algorithm is essentially the grayscale quantization of image, and its central idea is as follows: $K$ points are first randomly selected as the initial cluster center from the element set to be processed, then the distance between each element in the set and the cluster center is calculated, and each element is put into the cluster with the shortest distance from it, thus a single clustering process is completed; subsequently, the mean of all the elements of each cluster is calculated, and this mean is used as the cluster center in the following clustering. Iterative computation is repeated for each element, and new cluster centers are constantly generated in the computation process, until the convergence condition is met or no new elements are redistributed, which indicates the end of the clustering.

Let the data to be clustered be $X=\left\{x_{i}\right\}, x_{i} \in R^{n}, i=1,2 \ldots N$, and divide it into $K$ categories $L_{1}, L_{2}, \ldots, L_{k}$, then the description of $\mathrm{k}$ means clustering algorithm is as follows:

Step 1: Randomly select $k$ initial cluster centers $\eta_{1}, \eta_{2}, \ldots, \eta_{k}$ from the given data set;

Step 2: Calculate the distance between the element $x_{i}$ and each cluster center, $d_{i j}=\left|x_{i}-\eta_{j}\right|, \mathrm{j}=1$, $2, \ldots \mathrm{K}$

Step 3: Put $x_{i}$ into the cluster $L_{N}$ which is the closest to it, before calculating the mean of all the elements of this cluster and using the mean as a new clustering center, $\eta_{N}=\frac{1}{n} \sum_{x_{i} \in L_{N}} X_{i}$, where $n$ is the number of elements in the cluster $L_{N}$.

Step 4: Repeat Step 2 and Step 3, until the completion of the clustering of all the elements in the data set.

It can be seen from the principle of k-means clustering algorithm that the quality of clustering depends entirely on the determination of cluster quantity and the selection of the initial cluster center. In order words, cluster quantity and the initial cluster center decide the convergence speed and clustering precision of k-means clustering algorithm.

2. The determination of cluster quantity and the selection of the initial cluster center

1) The determination of cluster quantity

Cluster quantity can directly influence encoding speed and the quality of decoded image: if cluster quantity is small, the number of elements in each cluster will be large, and the scope of the search for the matching block for $R$-block will be large, thus the implementation duration will be long, though the quality of the reconstructed image will be good; on the other hand, a large cluster quantity will mean a small number of elements in each cluster, thus the search scope will be narrow and accordingly the encoding duration will be short, though the quality of the constructed image will be relatively poor and the duration of the clustering of the element sets will be relatively long, given that similar matching blocks are distributed to different clusters. Table 1 shows the PSNRs and encoding durations during the encoding of certain image in the presence of different $k$ values.

Table 1 The PSNRs and encoding durations for different $\mathrm{k}$ values

\begin{tabular}{lllllll}
\hline $\mathrm{K}$ & 2 & 3 & 4 & 5 & 6 & 7 \\
\hline $\mathrm{PSNR} / \mathrm{db}$ & 32.25 & 32.13 & 31.01 & 29.56 & 28.32 & 27.61 \\
$\mathrm{t} / \mathrm{s}$ & 74.31 & 67.58 & 55.48 & 38.42 & 35.53 & 35.26 \\
\hline
\end{tabular}


The above table indicates that it is not advisable to shorten the encoding duration by enlarging the cluster number. After comprehensively considering the quality of the reconstructed image and the encoding speed, empirical value $\mathrm{k}=4$ is selected in this paper.

2) The selection of the initial cluster center

According to the above analysis of k-means clustering algorithm's principle, it is crucial to situate the initial cluster center in the center area with intensely distributed elements, so that the elements around the center can be easily classified into the nearest clusters, resulting in the faster convergence of the clustering algorithm and a smaller number of iterations. Since analysis of the characteristic of random element distribution reveals the high probability of the clustering point being close to the mean of the cluster elements, and considering that standard deviation is an important parameter for evaluating element distribution, the standard deviation-mean method proposed in Literature (Wang Xiang-Yang et al, 2008) is used herein for selecting the initial cluster center.

Let the standard deviation of all data sets be $\rho$ and the mean be $\delta$, then the data should be scattered within the range $(\delta-\rho, \delta+\rho)$. If the number of clusters is $M, M$ equal-diversion points can be chosen in the range $(\delta-\rho, \delta+\rho)$ to constitute the initial cluster center during clustering. If the initial center of the $i$ th cluster is $L_{i}$, then:

$L_{i}=(\delta-\rho)+\frac{2 \rho}{L}, i=1 \ldots M$

In this paper, the standard deviation-mean method proposed in Literature (Wang Xiang-Yang et al, 2008) is integrated into the fractal image coding algorithm, and $R$-blocks and $D$-blocks are classified, with the purpose of narrowing the scope of the search for the matching blocks; moreover, the nearest neighbor search algorithm, instead of the global search method, is used for enhancing the speed of fractal coding.

3. The improved fast fractal coding method based on k-means clustering

Before the clustering operation for $R$-blocks and $D$-blocks, $R$-blocks are divided here into simple subblocks $\left(R_{\mathrm{var}}<=5\right)$ and complex bocks $\left(R_{\mathrm{var}}>5\right)$ according to their variances, in order to increase the encoding speed. In this paper, only complex $R$ blocks are encoded, k-means clustering algorithm is used for the classification of complex $R$-blocks, and the classification numbers of all the R-blocks are stored, before the nearest neighbor search method is employed to narrow the scope of the search for the matching blocks. The specific algorithm is as follows:
Step 1: Segment the image to be processed into non-overlapping $B \times B$ fixed blocks, namely, $R$ blocks;

Step 2: With $\lambda$ as step length, scan the image from left to right and from top to bottom, thus segment the image into $2 B \times 2 B D$-blocks which can be overlapped; then, use the method of fourneighborhood pixel averaging to process $D$-bocks so as to obtain image blocks which have the same sizes with the $R$-blocks, before performing eight kinds of isometric transformations to form the codebook.

Step 3: Calculate the variances of all the $R$ blocks, and divide the $R$-blocks into simple subblocks $\left(R_{\mathrm{var}}<=5\right)$ and complex sub-bocks $\left(R_{\mathrm{var}}>5\right)$. For simple sub-blocks, which needn't be encoded, directly store the coordinates of their upper left corners, calculate the mean of their pixel values and store the data.

Step 4: For complex sub-blocks, set the cluster number $k=4$, select the initial cluster center according to the standard deviation-mean method, perform $\mathrm{k}$-means clustering, and meanwhile record the new cluster centers generated after the iterations (see Section 4.1).

Step 5: Set the cluster number $k=4$, and use the final $R$-block cluster center as the initial cluster center for k-means clustering of $D$-blocks.

Step 6: For complex $R$-blocks to been coded, set the scope of the nearest neighbor search (see Section 3 ), search only this scope for the matching $D$-blocks of the same type; if $D$-blocks of the same type are not founded within this scope, search the domain of $D$-blocks of the same type outside this scope, and record five parameters of the best matching block: coordinates $(x, y)$ of its upper left corner, isometric transformation parameter $n$, grayscale translation parameters and contrast adjustment parameter $o$.

The decoding process is as follows: directly read the coordinates of the simple sub-blocks and the mean of the pixel values, and then, directly fill the mean pixel value in the corresponding position of the decoded image; according to the stored codebook parameters of the complex sub-blocks, find the information of $D$-blocks that each complex subblock corresponds to; perform multiple iterations by following the formula $\mathrm{R}=s \cdot D+o$ to restore the complex sub-blocks. Through about eight iterations, the original image can be restored based on a random image of the same size.

\section{ANALYSIS OF EXPERIMENTAL RESULTS}

In order to verify the superiority of this Algorithm, tests were conducted on 256x256x8bit standard grayscale images Lena, Peppers, Plane and Parrot by using Matlab7.0 on a Pentium (R) Dual-Core,2G- 
memory computer, and the results were compared with those for the basic fractal coding algorithm and for the algorithm put forward in Literature (Wang Xiang-Yang et al, 2008). In the experiment, the size of $R$-blocks was $4 \mathrm{x} 4$, the size of $D$-blocks was $8 \times 8$, and the step length $\lambda$ was 8 . The constructed images that the two standard test images correspond to, reconstructed separately by using the three algorithms, are as shown in Figure 5.

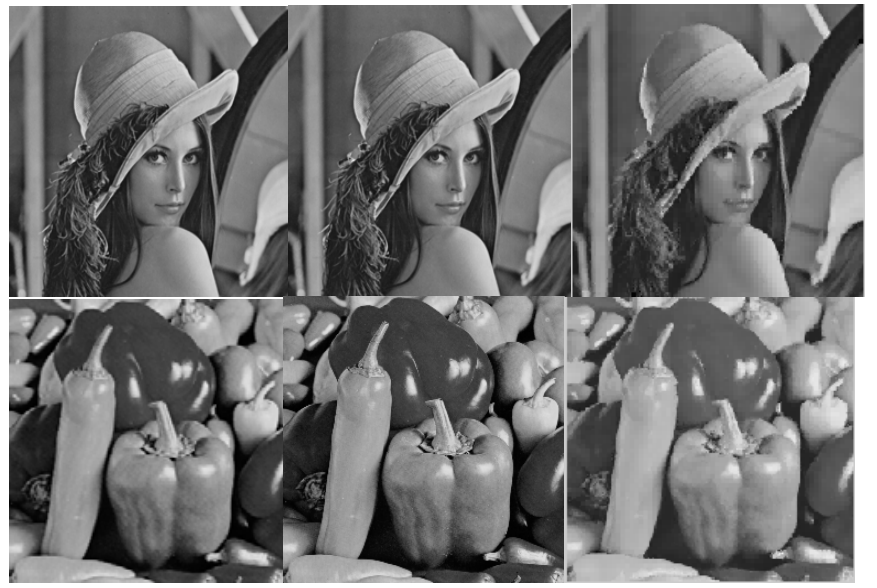

Fig. 5 The comparison of the images reconstructed using the three algorithms

According to Figure 5, the reconstructed images appear basically identical, which demonstrates the feasibility of this Algorithm. In the basic fractal coding algorithm and the algorithm from Literature (Wang Xiang-Yang et al, 2008), the compression ra- tio $C$ is fixed due to the fixed division of $R$-blocks and $D$-blocks, and the calculation formula for it is: $C=256 \times 256 \times 8 /(H \times(8+8+3+5+7)) \quad$ In this formula, $x$ and $y$ represent the coordinates of the upper left corner of $D$-block, respectively quantified as 8bit; $t$ represents the serial number of isometric transformation, quantified as $3 \mathrm{bit}$; $s$ represents the contrast adjustment factor, quantified as $5 \mathrm{bit}$; and $o$ represents the brightness adjustment factor, qualified as 7 bit. When $R$-block size is set to $4 \times 4, R$-block number is $H=256 / 4 \times 256 / 4=4096$, and the compression ratio is $C=4.13$. In the application of this Algorithm, the numbers of simple and complex subblocks are different. Let the total number of simple sub-blocks be $S_{l}$ and the total number of complex sub-blocks be $S_{2}$, then the calculation formula for the compression ratio $C$ is: $C=256 \times 256 \times$ $8 /\left(S_{1}+(1+8)+S_{2} \times(7+7+3+5+7)\right)$. Simple sub-blocks can be marked with 0 and quantified as 1 bit, the mean pixel value is quantified as 8 bit, the coordinates ( $x$ and $y$ )of the upper left corner of $D$-block are both quantified as $7 \mathrm{bit}$, and other parameters are consistent with those in the traditional algorithm. Table 2 shows the experiment data obtained when respectively using the basic fractal coding algorithm, the algorithm from Literature (Wang Xiang-Yang et al, 2008) and this Algorithm for testing the four standard images: encoding duration $T$ (unit: $S$ ), PSNR (unit: $d B$ ), compression ratio $C$ and speed-up ratio.

Table 2 The performance comparison among the three algorithms

\begin{tabular}{|c|c|c|c|c|c|c|}
\hline Image & $\begin{array}{c}\text { Performance } \\
\text { parameter }\end{array}$ & $\begin{array}{l}\text { The basic } \\
\text { algorithm }\end{array}$ & $\begin{array}{c}\text { Algorithm from } \\
\text { Literature (Wang } \\
\text { Xiang-Yang et al, } \\
\text { 2008) } \\
\end{array}$ & $\begin{array}{c}\text { This Algo- } \\
\text { rithm }\end{array}$ & $\begin{array}{l}\text { Speed-up ratio comparison } \\
\text { with the algorithm from } \\
\text { Literature (Wang Xiang- } \\
\text { Yang et al, 2008) }\end{array}$ & $\begin{array}{l}\text { Speed-up ratio } \\
\text { comparison with } \\
\text { the basic algorithm }\end{array}$ \\
\hline \multirow{4}{*}{ lena } & $T / s$ & 3038.33 & 1151.50 & 9.45 & \multirow{4}{*}{121.85} & \multirow{4}{*}{321.52} \\
\hline & $P S N R / d b$ & 31.19 & 30.57 & 30.00 & & \\
\hline & $C$ & 4.74 & 4.74 & 6.62 & & \\
\hline & $T / s$ & 2919.56 & 973.17 & 10.23 & & \\
\hline \multirow[t]{3}{*}{ Peppers } & $P S N R / d b$ & 32.61 & 30.66 & 30.08 & \multirow[t]{3}{*}{95.13} & \multirow[t]{3}{*}{285.39} \\
\hline & $C$ & 4.74 & 4.74 & 5.81 & & \\
\hline & $T / s$ & 2964.82 & 1035.88 & 7.76 & & \\
\hline \multirow[t]{3}{*}{ plane } & $P S N R / d b$ & 35.77 & 30.23 & 29.64 & \multirow[t]{3}{*}{133.49} & \multirow[t]{3}{*}{382.06} \\
\hline & $C$ & 4.74 & 4.74 & 26.69 & & \\
\hline & $T / s$ & 2967.76 & 989.25 & 5.20 & & \\
\hline \multirow[t]{2}{*}{ parrot } & $P S N R / d b$ & 33.59 & 30.89 & 29.82 & \multirow[t]{2}{*}{190.24} & \multirow[t]{2}{*}{570.72} \\
\hline & C & 4.74 & 4.74 & 11.91 & & \\
\hline
\end{tabular}

According to the data in Table 2, compared with the basic fractal coding algorithm, this Algorithm leads to slightly lower PSNR yet significantly shorter encoding duration - the maximum speed-up ratio can be up to 570 times as large; compared with the algorithm from Literature (Wang Xiang-Yang et al, 2008), this Algorithm leads to insignificantly lowered PSNR yet greatly reduced encoding durationthe maximum speed-up ratio is up to 190 times as large. Compared with the prior two algorithms, this Algorithm also leads to a higher compression ratio. A larger $R_{\text {var }}$ value means more simple sub-blocks, resulting in a higher compression ratio as well as slightly poorer quality of the decoded image due to the substitution of the mean pixel value for the original pixel values during the decoding of simple subblocks. The $R_{\text {var }}$ value is adjustable according to the actual need. 


\section{CONCLUSION}

In this paper, in view of the long duration of the search for matching blocks in fractal image coding algorithm, a variance-based classification method is applied to divide the sub-blocks into simple and complex sub-blocks, and only complex sub-blocks are encoded; then, the method proposed in Literature (Wang Xiang-Yang et al, 2008) is applied to select the initial cluster center and cluster the sub-blocks and parent blocks; and the nearest neighbor search method is applied to the process of searching for matching parent blocks, so as to match corresponding sub-blocks only with the neighboring parent blocks of the same type. The feasibility and advantage of this Algorithm have been verified by experimental results: while maintaining a PSNR of around 30, this Algorithm leads to a high encoding speed, which is more than 570 times larger than that of the algorithm proposed in Literature (Wang Xiang-Yang et al, 2008), and a better compression ratio.

\section{ACKNOWLEDGMENTS}

This work was supported by National Natural Science Foundation of China (Grant No. 61362038, Grant No. 61502343) and Guangxi Natural Science Foundation Program (Grant No. 2013GXNSFBA 019275, Grant No. 2013GXNSFBA019276, Grant No. 2015GXNSFAA139295)

\section{REFERENCES}

K.Jaferzadeh, K.Kiani, S.Mozaffari: Acceleration of fractal image compression sing fuzzy clustering and discretecosine-transform-based Metric. Image Processing, Vol.6 (2012) No.07, p.1024-1030.

Li Gaoping: Fast fractal image encoding algorithm based on three-mean feature. Journal of Image and Graphics, Vol.16 (2011) No.01, p.1-7.

Li Gaoping, Xiang Huifen, Zhao Zhengwu: Fast fractal image encoding algorithm based on quartiles feature, Computer Engineering and Applications,Vol.47(2011)No.22,p.145148.

Li Gaoping, Yang Jun, Chen Yihong: Fast fractal image encoding algorithm based on improved moment of inertia feature. Computer Engineering and Applications, Vol.49 (2013) No.24, p.144-148

Sheng Fei: Fast Encoding Algorithms for Fractal Image Compression (Ph.MS., Nanjing University of Posts and Telecommunications. China 2013), p.13-23.

Yang Pei: Research into Fast Search Method Based on Fractal Image Coding (Ph.MS., Nanjing University of Posts and Telecommunications. China 2013), p10-25.

Yi Chuanjun, Liu Liang:Fractal Image Encoding Using Edge Classification and Mean Deviation Comparison. Computer Application and Software. Vol.32 (2015) No.2, p.211-214.
Y.-L.Lin and M.-S.Wu: An edge property-based neighborhood region search strategy for fractal image compression. Computers \& Mathematics with Applications, Vol.62 (2011) No.01, p.310-318.

Wang Qiang, Liang De-qun, Bi Sheng: Nearest Neighbor Search for Fast Fractal Image Encoding Based on Correlation Information Feature. Journal of Chinese Computer Systems, Vol32 (2011) No.6, p.1108-1112.

Wang Xiang-Yang, Yu Yan-Chun: A Fast Fractal Image Compression Using the Improved K-mean Clustering. Computer Science, Vol. 35(2008) No.2, p.219-222. 